\title{
PROJECTIVE MODULI AND MAXIMAL SPECTRA OF CERTAIN QUOTIENT RINGS $\left({ }^{1}\right)$
}

\author{
BY
}

\begin{abstract}
ARON SIMIS
ABSTRACT. The projective modulus of a (commutative) ring is defined and a class of quotient rings is given for which the projective moduli are arbitrarily smaller than the dimension of the maximal spectra. Families of prime ideals of Towber and maximal type are introduced herein.
\end{abstract}

Introduction. The projective modulus of a commutative ring $A$, denoted proj $\bmod A$, is the least nonnegative integer $k$ (or $\infty$ ) such that every finitely generated projective $A$-module with well-defined rank is the direct sum of a free module and a module of rank $\leq k$. The fundamental result in this context is Serre's theorem [9] which asserts that if $A$ is noetherian then proj $\bmod A \leq \operatorname{dim} \max A$, where $\operatorname{dim} \max A$ stands for the (combinatorial) dimension of the maximal spectrum of $A$. Serre's theorem has in turn been greatly generalized by Bass [2, Chapter IV, \$2l. There is however a general lack of examples as to how the two invariants differ.

The purpose of this paper is to describe a general method of obtaining rings whose projective modulus is arbitrarily smaller than the dimension of their maximal spectrum. One of the main results proved herein is the following

Proposition 1.1. Let $A$ be a domain whose prime spectrum is a noetherian space of finite dimension and let. $A^{\prime}$ be a domain containing $A$ which is free as a $A$-module with basis of the form $1, \theta, \cdots, \theta^{d-1}$ for some $d \geq 1$. Let $\left\{P_{a}\right\}$ be a family of unramified prime ideals of $A$ such that, for every $\alpha$, there is a unique prime ideal $P_{a}^{\prime}$ of $A^{\prime}$ lying over $P_{a}$. If $S=A-\bigcup P_{a}$ and $S^{\prime}=A^{\prime}-\bigcup P_{a}^{\prime}$ then proj $\bmod \left(S^{\prime}\right)^{-1} A^{\prime} \leq(1 / d) \operatorname{dim} \max \left(S^{\prime}\right)^{-1} A^{\prime}$.

The prime spectrum of a ring is usually easier to treat than its maximal spectrum; it is the dimension of the latter however that is a sharper invariant. One would thus like, in the above proposition, to replace the condition on the prime

Received by the editors September 28, 1971.

AMS 1969 subject classifications. Primary 1340; Secondary 1320.

Key words and phrases. Projective modulus, maximal spectrum, families of prime ideals of Towber type, maximal type, unimodular element.

(1) These results are contained in the author's doctoral dissertation written under the guidance of Profess or P. Ribenboim at Queen's University, Kingston, Canada. The author was supported by the Canada Council. 
spectrum by a condition on the maximal spectrum. It turns out that to relate the maximal spectra of $A$ and $S^{-1} A$ is not so simple. This motivated and led me to consider families of maximal type: these are families $\left\{P_{a}\right\}$ of prime ideals of a ring $A$ such that if $S=A-\bigcup P_{a}$ then every maximal ideal of $S^{-1} A$ is of the form $S^{-1} P_{a}$ for some $\alpha$. This is classically the case for finite families of prime ideals and is a nice exercise; not so for infinite families. Thus I give some examples of infinite families of maximal type. As a bonus of these considerations, assuming the families of prime ideals to be of maximal type, I obtain (cf. Proposition 3.10) that the rings of Proposition 1.1 have homeomorphic maximal spectra. This homeomorphism had been obtained in [6] and [10] for particular cases and was in fact used to prove the analog of Proposition 1.1.

Families of maximal type come about only to a certain extent for the study of the maximal spectrum of $S^{-1} A$ in its relation to the maximal spectrum of $A$. The whole question is certainly far from being settled; undesired situations can occur at the very first examples. On the other hand, one may try to characterize those rings for which all families of prime ideals are of maximal type: this is the case for Dedekind domains with torsion class group (cf. Proposition 3.9).

I will also consider families of Towber type: these are families of prime ideals $\left\{P_{\alpha}\right\}$ of a ring $A$ satisfying the condition that for any two elements $f, g \in$ $\bigcup P_{a}$, there exists a nonconstant monic polynomial $q(T) \in A[T]$ (depending on $f$ and $g$ ) such that $q(f / g) \notin P_{a} A_{g}$ for every $\alpha$ for which $g \notin P_{\alpha}\left(A_{g}\right.$ stands for the ring of fractions of $A$ with respect to the powers of the element $g$ ). It is shown that, for a noetherian ring, families of Towber type are of maximal type. It is also shown that the families of prime ideals considered both in [6] and [10] are of Towber type.

The present work grew out of a correspondence with Lissner who suggested developing an idea of Towber. I have recently been informed by Lissner that he has a joint work with $\mathrm{K}$. L $\phi$ nsted which may imply some of my results; until the moment of finishing this paper, however, I had no knowledge of the content of theirs.

1. In this section I will prove the first of the propositions mentioned in the Introduction. The following result will be used:

Lemma 1.2. Let $A$ be a domain and let $A^{\prime}$ be a commutative A-algebra which is a free A-module of finite rank. Let $\left\{P_{a}\right\}$ be a family of prime ideals of $A$ such that, for every $a$, there exists a unique prime ideal $P_{a}^{\prime}$ of $A^{\prime}$ lying over $P_{a^{\prime}}$ set $S=A-U P_{a^{\prime}} S^{\prime}=A^{\prime}-\bigcup P_{a}^{\prime}$. Then $\left(S^{\prime}\right)^{-1} A^{\prime}=S^{-1} A^{\prime}$.

Proof. Since $S \subset S^{\prime}$, it is equivalent to show that the image of an element $s^{\prime} \in S^{\prime}$ along the canonical injection $A^{\prime} \rightarrow S^{-1} A^{\prime}$ is a unit. By $[3, \S 12$, no. 3 , 
Proposition 12l, one is to show that the norm of $s^{\prime}$ as an element of $S^{-1} A^{\prime}$ is a unit of $S^{-1} A$; in other words, one is reduced to proving that the norm of $s^{\prime}$ does not belong to $P_{a}$ for all $a$. On the other hand, the fact that there is a unique prime ideal $P_{\alpha}^{\prime}$ of $A^{\prime}$ lying over $P_{a}$ easily implies that $A_{P_{\alpha}^{\prime}}^{\prime}=A_{P_{\alpha}^{\prime}}^{\prime}$ (cf., e.g., [7, Chapter 2]). One may therefore assume that $A$ and $A^{\prime}$ are local and the structural morphism $A \rightarrow A^{\prime}$ is local; the result now follows from another application of $[3, \$ 12$, no. 3 , Proposition 12].

If $A$ is a ring and $M$ is an $A$-module, an element $x \in M$ is said to be $A$-unimodular if the $A$-homomorphism $A \rightarrow M$ defined by $a \rightarrow a x$ splits, i.e. if there exists an $A$-homomorphism $M \rightarrow A$ mapping $x$ onto 1 . In this case, $M \simeq A \oplus N$ for some $A$-module $N$.

Recall the fundamental result of the stable theory of projective modules:

Theorem 1.3 (Serre [9], Bass [2]). Let $A$ be a ring whose maximal spectrum is a noetherian space of dimension $r<\infty$. If $M$ is a finite projective A-module such that $\operatorname{rank}(M)>r$ at each point of $\max A$, then $M$ contains an A-unimodular element.

Let me now proceed to the

Proof of Proposition 1.1. Let $M^{\prime}$ be a finitely generated projective $\left(S^{\prime}\right)^{-1} A^{\prime}$ module such that $\operatorname{rank}\left(M^{\prime}\right)>r / d$, where $r=\operatorname{dim} \max S^{-1} A$. Let $M$ denote the underlying structure of $S^{-1} A$-module of $M^{\prime}$. By Lemma $1.2,\left(S^{\prime}\right)^{-1} A^{\prime}=S^{-1} A^{\prime}$ and, on the other hand, $S^{-1} A^{\prime}$ is a free $S^{-1} A$-module of rank $d$ by a base change argument. Therefore, since the rank of a module over a domain is the maximal number of linearly independent elements in the module, one easily deduces that $\operatorname{rank}(M)>$ $r$. It then follows from Theorem 1.3 that $M$ has an $S^{-1} A$-unimodular element, say $x \in M^{\prime}$. Let me show that $x$ is also $S^{-1} A^{\prime}$-unimodular: the argument is essentially due to Lissner and Moore [6], mine is a mere adaptation to the present general context.

Thus let $f: M^{\prime} \rightarrow S^{-1} A$ be an $S^{-1} A$-homomorphism such that $f(x)=1$. Define an $S^{-1} A$-homomorphism $g: M^{\prime} \rightarrow S^{-1} A^{\prime}$ by letting

$$
g(y)=\sum_{j=0}^{d-1} \sum_{i=0}^{d-1-j} a_{i+j+1} f\left(\theta^{j} y\right) \theta^{i}
$$

for $y \in M^{\prime}$, where $\theta^{d}=-a_{0}-a_{1} \theta-\cdots-a_{d-1} \theta^{d-1}$ for some $a_{0}, \cdots, a_{d-1} \epsilon$ $A$. The proof that $g$ is also $S^{-1} A^{\prime}$-linear proceeds exactly as in [6] and will not be repeated here. Now set $g(x)=a^{\prime} / s$, with $a^{\prime} \in A^{\prime}$ and $s \in S$. In order to show that $g(x)$ is a unit in $S^{-1} A^{\prime}=\left(S^{\prime}\right)^{-1} A^{\prime}$ it suffices to show that $a^{\prime} \notin P_{a}^{\prime}$ for every $\alpha$. Again as in [6] one sees that $a^{\prime} / s=\theta^{d-1}+\sum_{j=0}^{d-2}\left(c_{j} / t\right) \theta^{j}$ or, multiplying by a common denominator $u \in S, u a^{\prime}=u \theta^{d-1}+\sum_{j=0}^{d-2} b_{j} \theta^{j}$, for some 
$b_{j} \in A$. Now, for a fixed $\alpha$, set $P_{\alpha}=P$ and $P_{\alpha}^{\prime}=P^{\prime}$ and let $k$ (resp. $k^{\prime}$ ) be the residue field at $P$ (resp. $P^{\prime}$ ). Because of the hypothesis on $P, k^{\prime}$ is obtained from $k$ by base change; in particular, one has that the residues of $1, \theta, \cdots$, $\theta^{d-1} \bmod P^{\prime} A_{P^{\prime}}^{\prime}$ form a $k$-basis of $k^{\prime}$. Since $u \not \equiv 0 \bmod P^{\prime} A_{P^{\prime}}^{\prime}$ (because $u \in S$ ) it then follows that $u a^{\prime} \not \equiv 0 \bmod P^{\prime} A_{P^{\prime}}^{\prime}$, in particular $a^{\prime} \notin P^{\prime}$.

From the definition of projective modulus one then has proj $\bmod \left(S^{\prime}\right)^{-1} A^{\prime} \leq$ $r / d=(1 / d) \operatorname{dim} \max S^{-1} A$. To conclude the proof of the proposition one has only to remark that the finiteness assumptions on the prime spectrum of $A$ are inherited by the maximal spectrum of $S^{-1} A$ and use the following

Lemma 1.4. Let $R \subset R^{\prime}$ be rings such that $R^{\prime}$ is integral over $R$. Then $\operatorname{dim} \max R^{\prime}=\operatorname{dim} \max R$.

Proof. The inequality $\operatorname{dim} \max R^{\prime} \leq \operatorname{dim} \max R$ had been proved in [10]. The whole proposition has been independently obtained by Ohm and Pendleton [8, Proposition 3.1 (ii)].

The following is an amusing corollary for which I could find no explicit reference in the literature.

Corollary 1.5. Let $A$ be a Dedekind domain and let $A^{\prime}$ be the integral clos. ure of $A$ in a finite algebraic extension of the quotient field of $A$. Let $\left\{P_{a}\right\}$ be the family of prime ideals of $A$ inert in $A^{\prime}$; set $S^{\prime}=A^{\prime}-\bigcup P_{a}^{\prime}$ where $P_{a}^{\prime}$ is the unique prime ideal of $A^{\prime}$ lying over $P_{a}$. If $A^{\prime}$ is a free $A$-module with basis $1, \theta, \cdots, \theta^{d-1}$ for some $d \geq 2$, then $\left(S^{\prime}\right)^{-1} A^{\prime}$ is a principal ideal domain.

Proof. By the Krull-Akizuki theorem $\left[4, \$ 2\right.$, no. 5] or otherwise, $A^{\prime}$ is a Dedekind domain and so is $\left(S^{\prime}\right)^{-1} A^{\prime}$. The result now follows from Proposition 1.1 .

2. I will briefly show that the results of [10]-consequently those of [6]-are a special case of the preceding section.

Let $B^{\prime}$ be a domain and let $B$ be a subring of $B^{\prime}$. Look at the evaluations of $B\left[X_{1}, \cdots, X_{n}\right]$ (resp. $B^{\prime}\left[X_{1}, \cdots, X_{n}\right]$ ) at points of the set $B^{n}=B \times \cdots \times B$; these are ring homomorphisms $v_{a}: B\left[X_{1}, \cdots, X_{n}\right] \rightarrow B$ (resp. $v_{\alpha}^{\prime}: B^{\prime}\left[X_{1}, \cdots, X_{n}\right]$ $\left.\rightarrow B^{\prime}\right)$ defined for each $a=\left(a_{1}, \cdots, a_{n}\right) \in B^{n}$ by setting $v_{a}(f)=f(a)$, where $f \in B\left[X_{1}, \cdots, X_{n}\right]$ (resp. $f \in B^{\prime}\left[X_{1}, \cdots, X_{n}\right]$ ). It is clear that $v_{a}$ is surjective; on the other hand, one easily ckecks that $B\left[X_{1}, \cdots, X_{n}\right] /\left(X_{1}-a_{1}, \cdots, X_{n}-a_{n}\right)$ $\simeq B$. Therefore the homomorphism

$$
B\left[X_{1}, \cdots, X_{n}\right] /\left(X_{1}-a_{1}, \cdots, X_{n}-a_{n}\right) \rightarrow B\left[X_{1}, \cdots, X_{n}\right] / \operatorname{ker} v_{a}
$$

induced by the identity homomorphism is bijective; this implies that $\operatorname{ker} v_{a}=$ 
$\left(X_{1}-a_{1}, \cdots, X_{n}-a_{n}\right)$. Similarly, $\operatorname{ker} v_{a}^{\prime}=\left(X_{1}-a_{1}, \cdots, X_{n}-a_{n}\right)$ (ideal in $B^{\prime}[X]$ ). More generally, let $P$ be a prime ideal of $B\left[X_{1}, \cdots, X_{n}\right]$ such that $P^{\prime}=$ $P B^{\prime}\left[X_{1}, \cdots, X_{n}\right]$ is prime. One can evaluate elements of $B\left[X_{1}, \cdots, X_{n}\right] / P$ (resp. $B^{\prime}\left[X_{1}, \cdots, X_{n}\right] / P^{\prime}$ ) at points of $V(P)=\left\{a \in B^{n} \mid f(a)=0\right.$ for all $\left.f \in P\right\}$. These evaluations, still denoted $v_{a}$ (resp. $v_{a}^{\prime}$ ), obey the same laws as the former $v_{a}, v_{a}^{\prime}$. For instance, $\operatorname{ker} v_{a}$ is the ideal of $B\left[X_{1}, \cdots, X_{n}\right] / P$ generated by the residues of $X_{1}-a_{1}, \cdots, X_{n}-a_{n}$.

Given $\emptyset \neq W \subset V(P)$, consider the following multiplicative sets:

$$
\begin{aligned}
S & =S(W)=\left\{f \in B\left[X_{1}, \ldots, X_{n}\right] \mid v_{a}(f) \neq 0 \text { for all } a \in W\right\}, \\
S^{\prime} & =S^{\prime}(W)=\left\{g \in B^{\prime}\left[X_{1}, \ldots, X_{n}\right] \mid v_{a}^{\prime}(g) \neq 0 \text { for all } a \in W\right\} .
\end{aligned}
$$

It is a straightforward checking that

$$
\begin{aligned}
S & =B\left[X_{1}, \cdots, X_{n}\right]-\bigcup \operatorname{ker} v_{a}, \\
S^{\prime} & =B^{\prime}\left[X_{1}, \cdots, X_{n}\right]-\bigcup \operatorname{ker} v_{a}^{\prime},
\end{aligned}
$$

where $a$ runs through $W$.

Proposition 2.1 (Lissner-Moore [6], Simis [10]). Let $B$ be a domain whose prime spectrum is a noetherian space of finite dimension and let $B^{\prime}$ be a domain containing $B$ free as a $B$-module with basis $1, \theta, \cdots, \theta^{d-1}$ for some $d \geq 1$. Let $W, S, S^{\prime}$ be as indicated above and $A^{\prime}=B^{\prime}\left[X_{1}, \cdots, X_{n}\right] / P^{\prime}$. Then proj $\bmod \left(S^{\prime}\right)^{-1} A^{\prime} \leq(1 / d) \operatorname{dim} \max \left(S^{\prime}\right)^{-1} A^{\prime}$.

Proof. According to Proposition 1.1 one has only to check that (1) the prime ideals $\operatorname{ker} v_{a}$ are all inert in $A^{\prime}$ and the only prime ideal of $A^{\prime}$ lying over ker $v_{a}$ is $\operatorname{ker} v_{a}^{\prime} ;(2)$ the assumptions on the prime spectrum of $B$ are inherited by $B\left[X_{1}, \cdots, X_{n}\right] / P$. The first assertion is indeed immediate since $\operatorname{ker} v_{a}^{\prime} \cap$ $B\left[X_{1}, \cdots, X_{n}\right]=\operatorname{ker} v_{a}$ and $\left(\operatorname{ker} v_{a}\right) B^{\prime}\left[X_{1}, \cdots, X_{n}\right]=\operatorname{ker} v_{a}^{\prime}$. As for the second assertion, it is well known that finiteness of the Krull dimension is preserved under polynomial extensions and homomorphic images; that noetherianess of the prime spectrum is also preserved is a result of Ohm and Pendleton [8].

Observation. It has been fundamental in $\$ 1$ to assume that $A^{\prime}$ is a free $A$ module with basis of the form $1, \theta, \cdots, \theta^{d-1}$ for some $d \geq 1$. Thus, it will be important to decide when this happens: I call this the "existence of cyclic basis" problem. In [10] I surveyed several situations where $A$ was normal and $A^{\prime}$ was the integral closure of $A$ in a finite algebraic field extension of its quotient field; in this setup, of course, the examples are not very many. However, the present work represents a considerable improvement over $[10]$ in the sense that $A^{\prime}$ is not assumed to be normal. Thus, $A^{\prime}$ can be any subring of the integral closure of $A$ (in a finite extension of its quotient field) provided it possesses a cyclic 
basis; in particular, any simple extension of a normal domain will do it (cf. [10]).

3. As I pointed out before, Proposition 1.1 is unsatisfactory in the sense that it involves a hypothesis on the prime spectrum of $A$ whereas the leading role is, in fact, played by the maximal spectrum of certain ring of quotients of $A$. The same is true of Proposition 2.1; here the situation is even more serious since, for a polynomial ring $R[X]$, if the maximal spectrum of $R[X]$ is a noetherian space of finite dimension then the prime spectrum of $R$ is a noetherian space of finite dimension [8, Proposition 2.8 (iii), (iv)].

For arbitrary multiplicative set $S$, the maximal spectra of $A$ and $S^{-1} A$ can be completely unrelated (for instance, let $A$ be local, let $t \in A$ be a nonunit lying in at most "a few" prime ideals and let $S$ be the multiplicative set generated by $t$ ). However, for certain multiplicative sets, especially the saturated ones, some relation may be expected to hold. For example, if $S$ is the complement of a finite union of prime ideals then at least $\operatorname{dim} \max S^{-1} A=0 \leq \operatorname{dim} \max A$ ! How far this relation remains valid for complements of arbitrary families of prime ideals is unknown to me. Naively, the source of troubles lies in three aspects: (1) passage to quotients may not behave well enough with respect to infinite intersections; (2) difficulty in showing that an ideal whose extension is an intersection of maximal ideals is itself an intersection of maximal ideals; (3) if $S=$ $A-\bigcup P_{a}$ then it may certainly happen that a maximal ideal of $S^{-1} A$ is not the extension of some $P_{a}$.

I wish in this section to consider the third of the above problems.

Proposition 3.1. Let $A$ be a (commutative) ring and let $\left\{P_{a}\right\}$ be any family of prime ideals of $A$. The following conditions are equivalent:

(i) Every maximal ideal of $S^{-1} A$, where $S=A-\bigcup P_{\alpha^{\prime}}$ is of the form $S^{-1} P_{\alpha}$ for some $\alpha$.

(ii) Any ideal of A maximal among those not intersecting $S=A-U P_{a}$ is some $P_{a}$.

(iii) The canonical injection $\max S^{-1} A \rightarrow \operatorname{spec} A$ factors through the inclusion $\left\{P_{a}\right\} \subset \operatorname{spec} A$.

Proof. Easy from the definitions.

A family of prime ideals satisf ying the above equivalent conditions will be herein designated a family of maximal type.

Proposition 3.2. Let $A$ be a ring and let $\left\{P_{a}\right\}$ be a family of maximal type. Set $S=A-\bigcup_{P_{a}}$. Then one bas

(i) The family $\left\{S^{-1} P_{a}\right\}$ is a family of maximal type of the ring $S^{-1} A$.

(ii) If moreover there is no inclusion relation among the $P_{a}$ 's then $S^{-1} P_{a}$ 
is a maximal ideal of $S^{-1} A$ for every $\alpha$; in particular, $\max S^{-1} \simeq\left\{P_{a}\right\}$.

Proof. (i) Let $T=S^{-1} A-\bigcup S^{-1} P_{a}$ and let $T^{-1} Q$ be a maximal ideal of $T^{-1}\left(S^{-1} A\right)$, with $Q \in \operatorname{spec} S^{-1} A$. Then $Q$ is maximal among the ideals of $S^{-1} A$ contained in $\bigcup S^{-1} P_{a}$. On the other hand, any maximal ideal of $S^{-1} A$ is of the form $S^{-1} P_{a}$ for some $a$ by assumption. Therefore $Q=S^{-1} P_{\alpha}$ for some $\alpha$, hence, for this $\alpha, T^{-1} Q=T^{-1}\left(S^{-1} P_{\alpha}\right)$.

(ii) Given $S^{-1} P_{a}$, let $M \in \max S^{-1} A$ contain $S^{-1} P_{a}$; by hypothesis, $M=$ $S^{-1} P_{a^{\prime}}$ for some $\alpha^{\prime}$. Therefore $P_{a} \subset P_{a^{\prime}}$, hence $P_{a}=P_{a^{\prime}}$ by assumption.

Families of maximal type behave fairly well under homomorphic images:

Proposition 3.3. Let $A$ be a ring and $N$ an ideal, let $\left\{P_{a}\right\}$ be a family of prime ideals such that $N \subset P_{a}$ for every $a$. If $\left\{P_{a}\right\}$ is of maximal type then $\left\{P_{a} / N\right\}$ is of maximal type. Conversely, if every ideal of $A$, maximal among the ideals included in $U P_{a}$, contains $N$ then $\left\{P_{a}\right\}$ is of maximal type if $\left\{P_{a} / N\right\}$ is.

Proof. Firstly, $f(S)=(A / N)-\bigcup\left(P_{\alpha} / N\right)$, where $f: A \rightarrow A / N$ is the canonical homomorphism. Next one has a commutative diagram

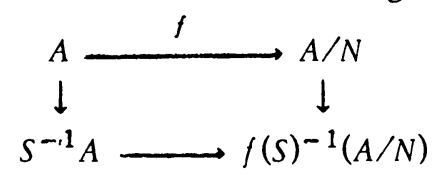

where the lower arrow $S^{-1} f: S^{-1} A \rightarrow f(S)^{-1}(A / N)$ is canonically defined by $a / s \mapsto f(a) / f(s)$. Now, if $Q \in \operatorname{spec} A$ is such that $N \subset Q$ then one obtains the following "key relation": $f(S)^{-1}(Q / N)=\left(S^{-1} f\right)\left(S^{-1} Q\right)$. Since $S^{-1} f$ is surjective, it follows that $S^{-1} Q \in \max S^{-1} A$ whenever one has $f(S)^{-1}(Q / N) \epsilon$ $\max \left(f(S)^{-1}(A / N)\right)$. If, moreover, $\left\{P_{a}\right\}$ is of maximal type then $S^{-1} Q=S^{-1} P_{a}$ for some $\alpha$, hence $Q=P_{a}$.

Conversely, suppose that every ideal of $A$, maximal among those contained in $\bigcup P_{\alpha}$, contains $N$. If $S^{-1} Q \in \max S^{-1} A$ one then has $N \subset Q$ and, from the "key relation", $f(S)^{-1}(Q / N)$ is a maximal ideal of $f(S)^{-1}(A / N)$. Since the family $\left\{P_{a} / N\right\}$ is assumed to be of maximal type, $f(S)^{-1}(Q / N)=f(S)^{-1}\left(P_{a} / N\right)$ for some a. Another use of the "key relation" now yields $\left(S^{-1} f\right)\left(S^{-1} Q\right)=\left(s^{-1} f\right)\left(S^{-1} P_{a}\right)$, hence $S^{-1} Q=S^{-1} P_{a}$.

Observation. It should perhaps be pointed out at this stage that the question whether a family of prime ideals is of maximal type is highly nongeometrical. To be precise, if $A$ is a ring and $E \subset A$ a subset, denote by $V(E)$ the set of prime ideals of $A$ containing $E$. If $I$ is the ideal generated by $E$, clearly $V(E)=$ $V(I)$. In particular, $V\left(\cup P_{a}\right)=V\left(\sum P_{a}\right)$ if $\left\{P_{a}\right\}$ is a family of prime ideals of $A$ and if the $P_{a}$ 's are comaximal then $V\left(\bigcup P_{a}\right)=V(A)=\emptyset$. Thus, in this case, knowing that $V(Q) \supset V\left(\bigcup P_{\alpha}\right)=\emptyset$ does not help to decide about $Q$ and the $P_{a}$ 's as sets. 
Since deciding whether a family is of maximal type can be very difficult, it is convenient to have a stronger concept which may be more accessible to check in practice.

Definition 3.4. Let $A$ be a ring and let $\left\{P_{\alpha}\right\}$ be a family of prime ideals of $A$. An ordered pair $(f, g)$ such that $f, g \in \bigcup P_{\alpha}$ is said to be a Towber pair with respect to $\left\{P_{a}\right\}$ if there exists a nonconstant monic polynomial $F(T) \in A[T]$ such that $F(f / g) \notin P_{a} A_{g}$ for every a for which $g \notin P_{a}$. The family $\left\{P_{a}\right\}$ itself is said to be of Towber type if every ordered pair $(f, g)$ such that $f, g \in \bigcup P_{a}$ is a Towber pair with respect to $\left\{P_{a}\right\}$.

Families of Towber type also behave as desired under homomorphic images. More precisely

Proposition 3.3 (bis). Let $A$ be a ring and $N$ an ideal, let $\left\{P_{a}\right\}$ be a family of prime ideals of $A$ such that $N \subset P_{a}$ for every a. A pair $(f, g)$ sucb that $f, g \in \bigcup P_{a}$ is a Towber pair with respect to $\left\{P_{a}\right\}$ if and only if the pair $(\bar{f}, \bar{g})$ is a Towber pair with respect to $\left\{P_{a} / N\right\}$, where $\bar{f}$ (resp. $\left.\bar{g}\right)$ denotes the residue of $f($ resp. $g) \bmod N$. In particular, $\left\{P_{a}\right\}$ is a family of Towber type if and only if $\left\{P_{a} / N\right\}$ is a family of Towber type.

Proof. Given $f, g \in A$ one has the following commutative diagram of ringhomomorphisms:



where the arrow $A[T] \rightarrow A_{g}$ (resp. $\left.(A / N)[T] \rightarrow(A / N)_{\bar{g}}\right)$ is evalutaion at $f / g$ (resp. $\bar{f} / \bar{g})$. The desired result easily follows by noting that $\left(P_{\alpha} / N\right)(A / N)_{\bar{g}} \simeq$ $P_{a} A_{g} / N A_{g}$ and by juggling with evaluations.

Corollary 3.4. Let $A$ be a ring and let $A_{\text {red }}$ be its reduced ring with the canonical surjection $f: A \rightarrow A_{\mathrm{red}}$. A family $\left\{P_{a}\right\}$ of prime ideals of $A$ is of maximal type (resp. Towber type) if and only if the family $\left\{f\left(P_{\alpha}\right)\right\}$ is of maximal type (resp. Towber type).

The next result establishes a connection between the above concepts.

Proposition 3.5. Let $A$ be a noetherian ring and let $\left\{P_{a}\right\}$ be a family of prime ideals of $A$. If $\left\{P_{\alpha}\right\}$ is of Towber type then it is of maximal type. 
Proof. Let $S^{-1} P$ be a maximal ideal of $S^{-1} A$, where $S=A-\bigcup P_{a}$. Since $A$ is noetherian, $P=\left(f_{1}, \cdots, f_{n}\right)$. The result is clearly true for $n=1$ without assuming the family $\left\{P_{\alpha}\right\}$ to be of Towber type. One proceeds by induction on $n$, using homogenization of polynomials. Thus, assume $n>1$ and let $F(T) \in A[T]$ be a nonconstant polynomial of leading coefficient 1 such that $F\left(f_{1} / f_{2}\right) \notin P_{a} A f_{2}$ for every $a$ for which $f_{2} \notin P_{a}$ (this exists by hypothesis); say, $F(T)=T^{k}+\cdots+$ $a_{0}$. Set $G\left(T_{1}, T_{2}\right)=T_{2}^{k} F\left(T_{1} / T_{2}\right)=T_{1}^{k}+\cdots+a_{0} T_{2}^{k}$. Clearly, $G\left(f_{1}, f_{2}\right) \in P$, so $G\left(f_{1}, f_{2}\right) \in P_{a}$ for some $a$. Since $P_{a} A_{f_{2}}$ is a prime ideal of $A_{f_{2}}$ it then follows that $f_{2} \in P_{\alpha}$. But $F(T)$ is monic, consequently also $f_{1} \in P_{\alpha}$ for the same $\alpha$.

It is now clear how to proceed: If $F_{1}(T) \in A[T]$ is a nonconstant monic polynomial for the Towber pair $\left(G\left(f_{1}, f_{2}\right), f_{3}\right)$, set $G_{1}\left(T_{1}, T_{2}, T_{3}\right)=$ $G\left(G\left(T_{1}, T_{2}\right), T_{3}\right)=G\left(T_{1}, T_{2}\right)^{j}+f_{3}(\ldots)$, where $j=$ degree of $F_{1}(T)$. Clearly one has $G_{1}\left(f_{1}, f_{2}, f_{3}\right) \in P$, so $G_{1}\left(f_{1}, f_{2}, f_{3}\right) \in P_{a^{\prime}}$ for some $\alpha^{\prime}$, hence $f_{3} \in P_{a^{\prime}}$ by an entirely similar argument as before. Since $F_{1}(T)$ is monic, $G\left(f_{1}, f_{2}\right) \in$ $P_{a^{\prime}}$ as well. From the induction hypothesis one then has $f_{1}, f_{2} \in P_{a^{\prime}}$.

At the outset, $P \subset P_{a}$ for some $\alpha$. Therefore $P=P_{a}$ for this $\alpha$ since $P$ is maximal among the ideals inside $\bigcup P_{a}$.

Corollary 3.6 (Towber, Simis). Let $D$ be a noetherian normal domain whose quotient field is not algebraically closed, let $I \subset D\left[X_{1}, \cdots, X_{n}\right]$ be an ideal and let $W$ be a nonempty subset of the "variety" of $I$ in $D^{n}=D \times \cdots \times D$. For $d \epsilon$ $W$, set $P_{d}=\operatorname{ker} v_{d}$ where $v_{d}: D\left[X_{1}, \cdots, X_{n}\right] / I \rightarrow D$ is evaluation at $d$. Then the family $\left\{P_{d}\right\}_{d \in W}$ is of maximal type.

Proof. By Proposition 3.5 it suffices to show that $\left\{P_{d}\right\}_{d \in W}$ is a family of Towber type. I will in fact prove something much stronger, namely, that there exists a "universal" polynomial for all pairs $(f, g)$ such that $f, g \in \bigcup P_{d}$.

First I claim that there exists a nonconstant monic polynomial in $D[T]$ having no roots whatsoever in the quotient field $K$ of $D$; for otherwise, it follows from the normality of $D$ that every monic polynomial over $D$ splits completely inside $D$. That is, if $D^{\prime}$ is the integral closure of $D$ in an algebraic closure $\bar{K}$ of $K$, then $D=D^{\prime}$, so $K=\vec{K}$ as well.

Next, it is enough to consider the case $I=(0)$ (cf. Proposition 3.3 (bis)). Let $f, g \in \bigcup P_{d}, d \in W$. If $d \in W$ is such that $g \notin P_{d}$ then $g(d)=v_{d}(g) \neq 0$, therefore $v_{d}(g)$ becomes a unit in the ring $D_{v_{d}(g)}$, hence $v_{d}$ extends to a homomorphism $v_{d}:\left(D\left[X_{1}, \cdots, X_{n}\right]\right)_{g} \rightarrow D_{v_{d}(g)}$. Let $F(T)=T^{k}+\cdots+d_{0} \in D[T]$ be chosen as above. Then $v_{d}(F(f / g))=v_{d}\left(f^{k} / g^{k}+\cdots+d_{0}\right)=\left(v_{d}(f) / v_{d}(g)\right)^{k}+$ $\cdots+d_{0}$ which implies $v_{d}(F(f / g)) \neq 0$ because $v_{d}(f) / v_{d}(g) \in D_{v_{d}(g)} \subset K$ and, by assumption, $F(T)$ has no roots in $K$. It follows that $F(f / g) \notin P_{d} A_{g}$, as required. 
It is a well-known fact that finite families of prime ideals in an arbitrary ring are of maximal type; this is because an ideal is not contained in a finite union of prime ideals unless it is contained in at least one of these prime ideals. An argument reminiscent of the one used to prove this result yields the following.

Proposition 3.7. Let $A$ be a ring and let $\left\{P_{i}, 1 \leq i \leq t\right\}$ be prime ideals of $A$ with no inclusion relation among themselves. Then $\left\{P_{i}, 1 \leq i \leq t\right\}$ is a family of Towber type.

Proof. Let $f, g \in \bigcup_{i=1}^{t} P_{i}$. One may clearly assume that $g \notin P_{i}$ for at least one $i$ (otherwise the Towber condition is emptily verified). Let $g \in P_{i}, \cdots, P_{s}$, $g \notin P_{s+1}, \cdots, P_{t}(s<t)$ and distinguish two cases:

(1) $f \in P_{s+1}, \cdots, P_{t}$. In this case the Towber condition for the pair $f, g$ is easily verified with $F(T)=T+g$ for instance.

(2) $f \notin P P_{j}$ for some $j$ such that $s+1 \leq j \leq t$. Rearrange the $P$ 's in such a way that $f \notin P_{s+1}, \cdots, P_{s+k-1}$ and $f \in P_{s+k}, \cdots, P_{t}$, with $k>1$. Now, for each $j=s+1, \cdots, s+k-1$ let $a_{j} \in A$ be such that $a_{j} \in P_{j}$ and $a_{j} \notin P_{s+k}$, $\cdots, P_{t}$ (this is possible by assumption). Set $F(T)=T+\Pi_{j=s+1}^{s+k-1} a_{j}$. One readily verifies that $F(f / g) \notin P_{s+1} A_{g}, \cdots, P_{t} A_{g}$.

Other examples of families of Towber type are obtained by looking at topological rings and rings of convergent series. More precisely, let $A$ be a topological ring whose topology is linear. Recall that this means there exists a fundamental system $\left(I_{\lambda}\right)$ of neighborhoods of 0 consisting of ideals. Suppose, moreover, that $A$ is separated and complete with respect to the given topology. Set

$$
A\left\{T_{1}, \cdots, T_{n}\right\}=\underline{\lim }\left(A / I_{\lambda}\right)\left[T_{1}, \cdots, T_{n}\right],
$$

where the $T$ 's are indeterminates; $A\left\{T_{1}, \cdots, T_{n}\right\}$ is a separated complete topological ring whose topology is linear and it can be identified with a subring of the formal power series $A\left[\left[T_{1}, \cdots, T_{n}\right]\right]$. Recall that $[5,7.5 .3]$ given $\left(a_{1}, \cdots, a_{n}\right) \in A^{n}$, there exists a unique (continuous) $A$-algebra homomorphism $f: A\left\{T_{1}, \cdots, T_{n}\right\} \rightarrow A$ such that $f\left(T_{i}\right)=a_{i}(1 \leq i \leq n)$. Moreover, if the $a_{i}$ 's are topologically nilpotent, there exists an $A$-algebra homomorphism $A\left[\left[T_{1}, \cdots, T_{n}\right]\right] \rightarrow A$ such that $T_{i} \mapsto a_{i}(1 \leq i \leq n)$.

Similarly, if $K$ is a complete nondiscrete valued field and $E$ a subset of $K^{n}=K \times \cdots \times K$, one may consider the subring $K\left\langle\left\langle T_{1}, \cdots, T_{n}\right\rangle\right\rangle_{E}$ of $K\left[\left[T_{1}, \cdots, T_{n}\right]\right]$ of convergent power series on $E$ (cf. $\left.[1, \S 2]\right)$. Then if $a \in E$, the evaluation $f \mapsto f(a)$ is a well-defined $A$-algebra homomorphism from $K\left\langle\left\langle T_{1}, \cdots, T_{n}\right\rangle\right\rangle_{E}$ to $K$.

In any case, one can also consider evaluations modulo an ideal. Furthermore, 
whenever evaluations of $A\left[\left[T_{1}, \cdots, T_{n}\right]\right]$ are concerned it is understood that they are defined relative to topologically nilpotent elements.

Proposition 3.8. Let $A$ (resp. K) be a noetherian normal domain equipped with a complete linear topology (resp. a complete nondiscrete valued field) such that the quotient field of $A$ (resp. K) is not algebraically closed. Let $R$ stand for either $A\left\{T_{1}, \cdots, T_{n}\right\}, A\left[\left[T_{1}, \cdots, T_{n}\right]\right]$ or else $K\left\langle\left\langle T_{1}, \cdots, T_{n}\right\rangle\right\rangle_{E}$ and let $I$ be an ideal of $R$. Let $W$ be a nonempty subset of the variety of $I$ in $A^{n}$ (resp. $E$ ) and for each $a \in W$ let $P_{a}=\operatorname{ker} v_{a}$, where $v_{a}: R / I \rightarrow A$ (resp. K) is the evaluation at $a$. Then the family $\left\{P_{a}\right\}_{a \in W}$ is of maximal type.

Proof. The proof is verbatim the proof of Proposition 3.6.

It is easy to see that an arbitrary family of prime ideals in a principal ideal domain is of maximal type. In fact, a more general result holds:

Proposition 3.9 (P. J. Cahen). Let $A$ be a Dedekind domain with torsion class group. Then an arbitrary family of prime ideals in $A$ is of maximal type.

Proof. Let $\left\{P_{v}\right\}$ be a family of prime ideals, where $v$ is a valuation associated with $P_{v}$. For a given $P_{v_{0}}$ not of this family, there exists, by hypothesis, a nonnegative integer $n=n\left(v_{0}\right)$ such that $P_{v_{0}}^{n}=A y$ for some $y \in A$. Then $v(y)=$ 0 for every $v \neq v_{0}$, hence $y \notin P_{v}$ for every $v \neq v_{0}$. In particular, if $S=A-\bigcup P_{v}$ then any nonzero prime ideal of $S^{-1} A$ is of the form $S^{-1} P$.

Let me turn now to the question of the homeomorphism between the maximal spectra of the rings $S^{-1} A$ and $\left(S^{\prime}\right)^{-1} A^{\prime}$ introduced in $\$ 1$. There is no apparent reason to conjecture that these spectra are homeomorphic in general. However one can prove the following result:

Proposition 3.10. Let $A$ be a domain and let $A^{\prime}$ be a commutative A-algebra which is a free A-module of finite rank. Let $\left\{P_{a}\right\}$ be a family of maximal type in $A$ and suppose that, for every $\alpha$, there exists a unique prime ideal $P_{\alpha}^{\prime}$ of $A^{\prime}$ lying over $P_{\alpha}$. Then the maximal spectra of $S^{-1} A$ and $\left(S^{\prime}\right)^{-1} A^{\prime}$ are bomeomorphic, where $S=A-\bigcup P_{a}$ and $S^{\prime}=A^{\prime}-\bigcup P_{\alpha}^{\prime}$.

Proof. By Lemma 1.2, it suffices to consider the rings $S^{-1} A$ and $S^{-1} A^{\prime}$. Since $\left\{P_{a}\right\}$ is of maximal type, a maximal ideal of $S^{-1} A$ is of the form $S^{-1} P_{a}$. Let $P_{a}^{\prime}$ be the unique prime ideal of $A^{\prime}$ lying over $P_{a}$; then, clearly, $S^{-1} P_{a}^{\prime}$ lies over $S^{-1} P_{a}$. If, moreover, $P^{\prime} \in \operatorname{spec} A^{\prime}$ is such that $S^{-1} P^{\prime}$ lies over $S^{-1} P_{a}$ then $S^{-1} P_{a}=\left(P^{\prime} \cap A\right) S^{-1} A=S^{-1}\left(P^{\prime} \cap A\right)$; therefore $P^{\prime}=P_{a}^{\prime}$. On the other hand, $S^{-1} A^{\prime}$ is integral over its subring $S^{-1} A$. The conclusion is now derived from the easy 
Lemma 3.11. Let $R \subset R^{\prime}$ be commutative rings with $R^{\prime}$ integral over $R$. If for every maximal ideal of $R$ there is one single maximal ideal of $R^{\prime}$ lying over it, then the maximal spectra of $R$ and $R^{\prime}$ are bomeomorphic.

Proof. The homeomorphism is in fact canonical; by integrality, the continuous mapping $f: \operatorname{spec} R^{\prime} \rightarrow \operatorname{spec} R$ defined by $P^{\prime} \mapsto P^{\prime} \cap R$ induces a continuous mapping $f_{\max }: \max R^{\prime} \rightarrow \max R$ which is a bijection by assumption. On the other hand, $f$ is a closed mapping since $R^{\prime} / I^{\prime}$ is integral over $R / I^{\prime} \cap R$ for every ideal $I^{\prime}$ of $R^{\prime}$. Therefore $f_{\max }$ is closed as well.

\section{REFERENCES}

1. S. S. Abhy ankar, Local analytic geometry, Pure and Appl. Math., vol. 14, Academic Press, New York, 1964. MR 31 \#173.

2. H. Bass, Algebraic K-theory, Benjamin, New York, 1968. MR $40 \# 2736$.

3. N. Bourbaki, Eléments de mathématique. XXIII. Part. 1. Les structures fondamentales de l'analyse. Livre II. Algèbre. Chap. 8: Modules et anneaux semisimples, Actualités Sci. Indust., no. 1261, Hermann, Paris, 1958. MR 20 \#4576.

4. - Eléments de mathématique. Fasc. XXX. Algèbre commutative. Chap. 5: Entiers, Actualités Sci. Indust., no. 1308, Hermann, Paris, 1964. MR 33 \#2660.

5. A. Grothendieck, Eléments de géométrie algébrique. I. Le language des schémas, Inst. Hautes Etudes Sci. Publ. Math. No. 4(1960). MR 36 \#177a.

6. D. Lissner and N, Moore, Projective modules over certain rings of quotients of affine rings, J. Algebra 15 (1970), 72-80. MR $41 \# 1715$.

7. H. Matsumura, Commutative algebra, Benjamin, New York, 1970.

8. J. Ohm and R. L. Pendleton, Rings with Noetherian spectrum, Duke Math. J. 35 (1968), 631-639. MR 37 \#5201.

9. J.-P. Serre, Modules projectifs et espaces fibrés à fibre vectorielle, Séminaire P. Dubreil, M.-L. Dubreil-Jacotin et C. Pisot, 1957/58, fasc. 2, Exposé 23, Secrétariat mathématique, Paris, 1958, MR 31 \#1277.

10. A. Simis, Projective modules of certain rings and the existence of cyclic basis, J. Algebra 18 (1971).

DEPARTMENT OF MATHEMATICS, QUEEN'S UNIVERSITY, KINGSTON, ONTARIO, CANADA 